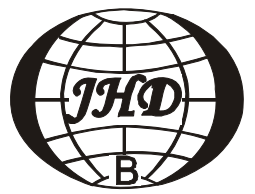

sdlj.chinajournal.net.cn

\title{
INVESTIGATION OF TURBULENCE STRUCTURES AND TURBULENT COUNTER-GRADIENT TRANSPORT PROPERTIES IN STRATIFIED FLOWS
}

\author{
Ph.D. Candidate: QIU Xiang \\ Shanghai University, Shanghai Institute of Applied Mathematics and Mechanics, Shanghai 200072, China, \\ E-mail: emqiux2008@yahoo.com.cn
}

\section{Supervisor:}

LIU Yu-lu(Shanghai University)

Members of Dissertation Defense Committee:

$\mathrm{Xu}$ You-heng(Fudan University), Chairman

Zhou Lian-di(CSSRC)

Dai Shi-qiang(Shanghai University)

Lu Chuan-jing(Shanghai Jiaotong University)

Liu Hua(Shanghai Jiaotong University)

Time of Dissertation Defense: October 16, 2006

\begin{abstract}
Turbulence structures and turbulent Counter-Gradient Transport(CGT) properties in the stratified flows with a sharp temperature interface are investigated by experimental measurements using LIF and PIV, by LES and by correlation analysis.
\end{abstract}

KEY WORDS: stratified turbulence, counter-gradient transport, coherent structure, Hilbert-Huang transform

BRIEF INTRODUCTION OF THE PAPER: In this article, the collective effects of mean shear and stratification on turbulence structure and turbulent scalar Counter-Gradient Transport(CGT) features are studied principally.

The set of experiments are conducted in neutral and stratified flows using LIF and PIV. The results show that, turbulent mixing is improved by mean shear but suppressed by stratification, the large-scale structures are restricted within the mixing layer, particularly in stratified case. In addition, very complicated phenomena of stretching, uniting and distorting between vortices are found nearby the interface, the remarkable revolving and anti-revolving vortex pairs and the finger-like structure are found.

LES is then applied to study stably stratified turbulence with a sharp interface. It shows that: the streamwise fluctuations are stronger than the vertical fluctuations, turbulent fluctuations decay more rapidly at the presence of stratification. The offset of the mixing layer to the side with small inlet velocity is attained quantitatively. The small-scale scalar structures decrease with the decrease of stratification. Turbulent scalar CGT is found at the central area of the flow with the range of $x / M<10$ in streamwise direction.

The times series of velocities and temperature of stratified turbulence are analyzed by Hilbert-Huang transform. It is found that: (1) Turbulent statistics depart from the Gaussian distribution at most of modes, velocity intermittency decreases along the down stream, and the intermittency is scattered to many more modes, the intermittency is intensified by the stratification, especially in small scales. (2) The flows show the anisotropy, coherent structures play a principal role in the stratified flows, the energy isdistributed to many more modes with the effects of stratification. (3) Hilbert spectrum shows that the energy of velocity fluctuation is concentrated in the low frequency, therefore the energy is mostly contained in the coherent structure. (4) There are local CGT phenomena at certain modes whereas Gradient Transport (GT) occurs at other modes, and the modes of coherent structures are strongly associated with the modes which contributes to principal part of scalar transport.

Lastly, scalar CGT properties are studied applying LES and correlation analysis comparatively in linear stably stratified turbulence. The suppressive effects of stratification are validated, and the wave number of the largest scale structure which contributes to scalar CGT is found by the correlation analysis, and the scale of the structure decreases with the enhancing of the stratification. 